\title{
Infalf.
}

Sinngebidte.

Seite

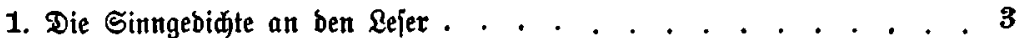

2. Ebendiejelben . . . . . . . . . . . . . . . . 3

3. Auf ben neuern Theil biejer Sinngebidte . . . . . . . . . . . . 3

4. Der Stadjelreim. . . . . . . . . . . . . . . . . 3

5. Mitander . . . . . . . . . . . . . . . . . . . 4

6. $\mathfrak{U}_{\mathfrak{n}}$ ben Marull . . . . . . . . . . . . . . . . . . . . 4

7. Merlur und $\mathfrak{A m o x}$. . . . . . . . . . . . . . . . . . . 4

8. Thrax und Star . . . . . . . . . . . . . . . . 4

9. Der geizige Difhter . . . . . . . . . . . . . . . . . . 5

10. $\mathfrak{X} u f$ Eucinden . . . . . . . . . . . . . . . . 5

11. Iuf bie Europa . . . . . . . . . . . . . . . . . . . 5

12. ßompils Landgut . . . . . . . . . . . . . . . . . 6

13. Wiberruf beş Borigen . . . . . . . . . . . . . . . . . 6

14. $\mathfrak{A n}_{\mathfrak{n}}$ bie berren $\mathfrak{X}$ und $\mathfrak{Y}$. . . . . . . . . . . . . . . . . 6

15. Die Emigleit gewiffer Bebiate . . . . . . . . . . . . . 6

16. $\mathscr{A u f ~ b a s ~ \Im u n g f e r n f t i f t ~} \mathfrak{u}^{*} *$. . . . . . . . . . . . . . 6

17. $\mathfrak{A n}$ ben Doftor $\mathfrak{S}_{\mathfrak{p}}^{* *}$. . . . . . . . . . . . . . . . . 7

18. Auf ben Mnemon . . . . . . . . . . . . . . . 7

19. Bavgి Baft . . . . . . . . . . . . . . . 7

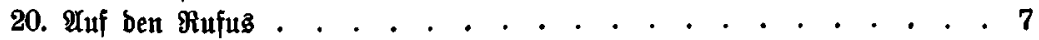

21. Iuf Dorinben . . . . . . . . . . . . . . . . 8

22. $\mathfrak{A} \mathfrak{n}$ bas Bilb ber Bereøtigleit, in bem

Antwort . . . . . . . . . . . . . . . 8

23. 2 uf einen abeligen Dummfopf . . . . . . . . . . . . . 8

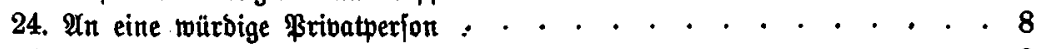

25. Aluf bie Эriß . . . . . . . . . . . . . . . . . . 8

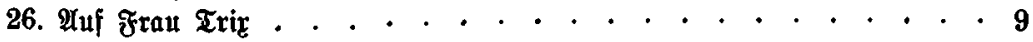

27. $\mathfrak{A} u \mathfrak{f}$ \&ufrins $\mathbb{S} \mathfrak{a} \mathfrak{a b}$. . . . . . . . . . . . . . . . . . 9

Leffing, fämtlide Sdutiften. I. 


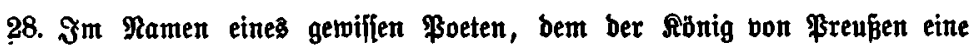
golbene Doje fhentite. . . . . . . . . . . . . . . . . 9

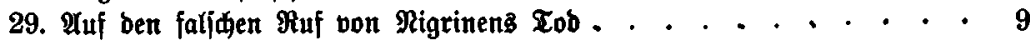

30. $\mathfrak{A} \mathfrak{u} \mathfrak{\text { ben }}$ Sargil . . . . . . . . . . . . . . . . . 9

31. Die .Ffudt . . . . . . . . . . . . . . . . . . . . 10

32. Die Mohlthaten. . . . . . . . . . . . . . . . . . . 10

33. . An einen Seizigen . . . . . . . . . . . . . . . . . . 10

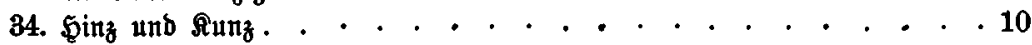

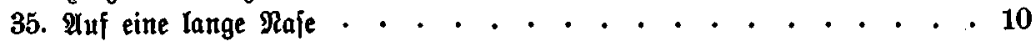

36. Iuf Stipjen . . . . . . . . . . . . . . . . . . . 11

37. IUf bett Santulus . . . . . . . . . . . . . . . . 11

38. $\mathfrak{A n}$ Grillen . . . . . . . . . . . . . . . . . . . . . 11

39. Un ben Salomon . . . . . . . . . . . . . . . . . 12

40. IIuf ebendenjelben . . . . . . . . . . . . . . . . 12

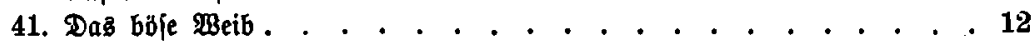

42. $\mathfrak{A n}$ ben $\mathfrak{A} e m i l$. . . . . . . . . . . . . . . . . 12

43. Trux an ben Sabin . . . . . . . . . . . . . . . . . . 12

44. Untwort bes Sabin . . . . . . . . . . . . . . . . . 13

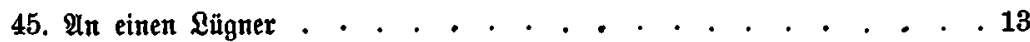

46. $\mathfrak{A}$ uf $\mathfrak{T r i l l}$ unb $\mathfrak{T r o l l}$. . . . . . . . . . . . . . . . 13

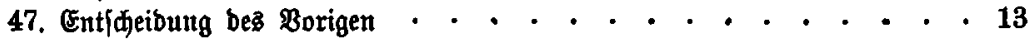

48. $\mathfrak{U n ~ b i e ~}^{*}$. . . . . . . . . . . . . . . . . . . . 13

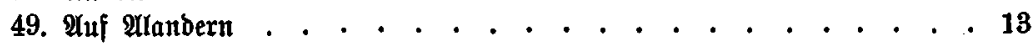

50. 9 Uf einen Brand zu * * . . . . . . . . . . . . . . . . . . 14

51. $\mathfrak{A n}$ cinen. . . . . . . . . . . . . . . . . . . . . 14

52. Brabjajrijt bes গitulus . . . . . . . . . . . . . . . 14

53. $\mathfrak{A} u f$ ben Sobthll ,. . . . . . . . . . . . . . . . . . . 14

54. Ân ben ßompil . . . . . . . . . . . . . . . . . . . 14

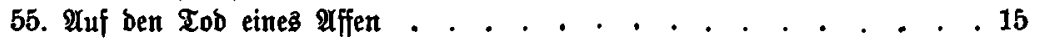

56. Srabjatrift auf ebendenjelben . . . . . . . . . . . . . . 15

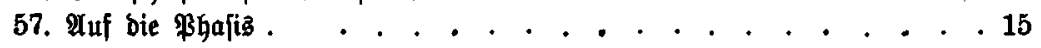

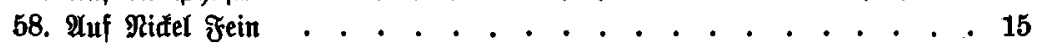

59. IUf eine Riebhaberinn bes Trauerppiés . . . . . . . . . . . 16

60. $\mathfrak{A}$ f ein Sdjladtftüł von Sugtenburg . . . . . . . . . . 16

61. Uuf ben Sablador . . . . . . . . . . . . . . . 16

62. Auf ben $\mathfrak{M i f o n}$. . . . . . . . . . . . . . . 16

63. Der reide Freber . . . . . . . . . . . . . . . . . 17

64. Uuf ben Mufinus . . . . . . . . . . . . . . . . . . 17

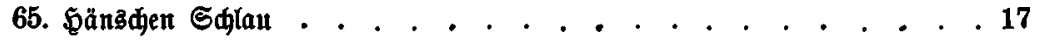

66. $\mathfrak{A n}$ bie Doriliz . . . . . . . . . . . . . . . . . . . . 17

67. Erabjdrift eines unglïdfliden, welder zuleşt in eirtem Sđiffbrudje umfam 17

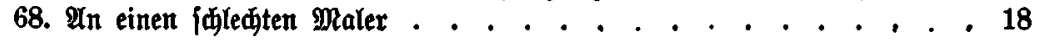


69. $\mathfrak{A}$ uf eine Bilbjäule beş $\mathfrak{A}$ mor $\ldots \ldots \ldots$

70. $\mathfrak{A} u \mathfrak{f}$ ebendiefelbe .

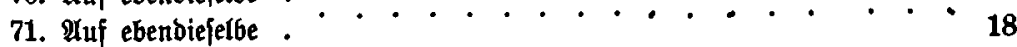

72. $\mathfrak{A} u \mathfrak{f}$ ebendiejelbe . . . . . . . . . . . . . . . 18

73. $\mathfrak{A} u f$ ebendiejelbe . . . . . . . . . . . . . . . 19

74. Auf ben ₹abull . . . . . . . . . . . . . . . 19

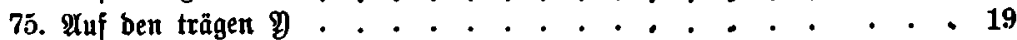

76. Entiøulbigung megen unterlaffenen Bejuðs . . . . . . . . . 19

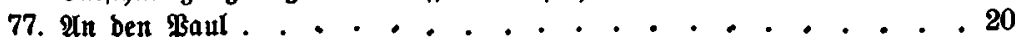

78. Belt und \$olt. . . . . . . 20

79. Der tranfe Star.

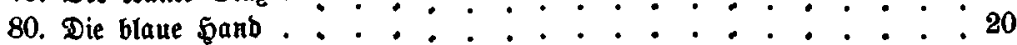

81. Der Sれufter franz. . . . . . . . . . . . . . . 21

82. Das Mäbđen. . 21

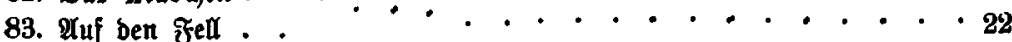

84. $\mathfrak{A n}_{\mathfrak{n}}$ ben $\mathfrak{J e r r n} \mathfrak{D}^{*} . . \quad$. . . . . . . . . . . . 22

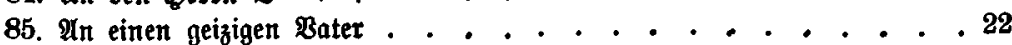

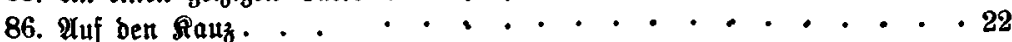

87. $\mathfrak{A} u f$ ben Supan ..... . . . . . . . . . . . . . 22

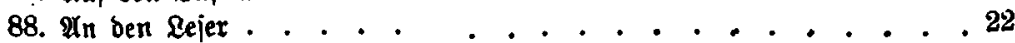

89. Un $_{\mathbf{n}}$ ben Serrn bon Dampf _. . . . . . 23

90. Int ebendenfelben. . . . • . . . • . . . . . . 23

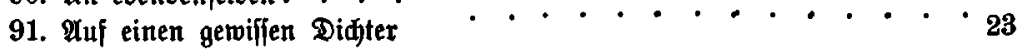

92. $\mathfrak{A}_{\mathfrak{n}}$ ben $\mathfrak{W}_{\text {eip }} \ldots \ldots, \ldots, \ldots$

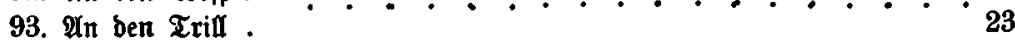

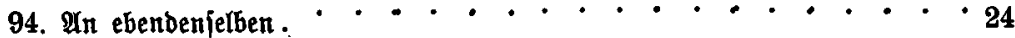

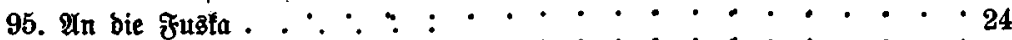

96. $\mathfrak{A}$ uf ben Iob bes $\mathfrak{D}$ Meab .

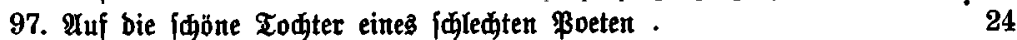

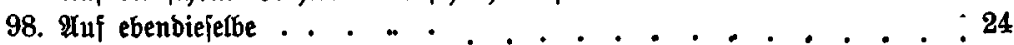

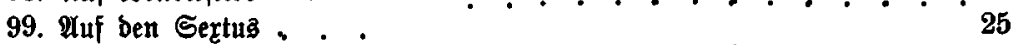

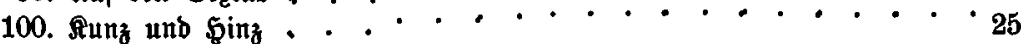

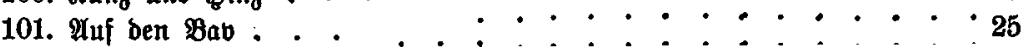

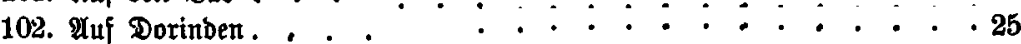

103. IUuf die Balathee. . . . . . . . . . . . . . . . 26

104. $\mathfrak{A}$ f bie Đütte beş łrus . . . . . . . . . . . . . . 26

105. $\mathfrak{A} u f$ einen gemiffen \&eidenrebner . . . . . . . . . . . 26

106. Das fdlimmite Thier . . . . . . . . . . . . 26

107. $\mathfrak{A} u f$ bie Magbaliz . . . . 26

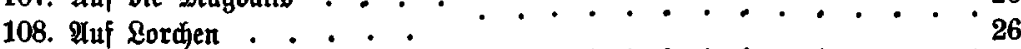

109. Rlimps . . . . . . . . •

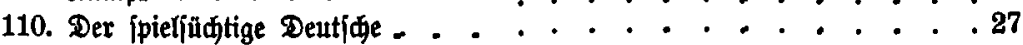




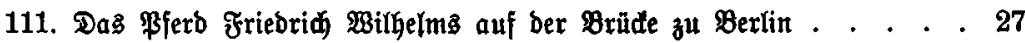

112. $\mathfrak{A} u f$ bie feige $\mathfrak{D}_{u n m a}$. . . . . . . . . . . . . . . . . 27

113. Gine Sefunbheit auf bie Gejunbgeiten. . . . . . . . . . . . 27

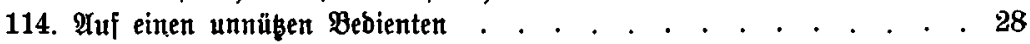

115. Der Sđtwux . . . . . . . . . . . . . . . . . . . . 28

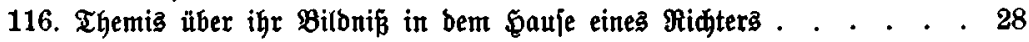

117. Der Furøtjame . . . . . . . . . . . . . . . . . . . 28

118. $\mathfrak{A n}$ ben Đerrn $\mathfrak{B}$. . . . . . . . . . . . . . . . . . . 29

119. Яนі die Benejung einer ßublerinn . . . . . . . . . . . . . 29

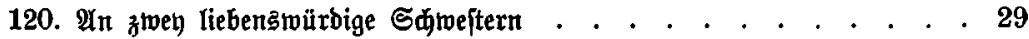

121. An ben Silius . . . . . . . . . . . . . . . . . . 29

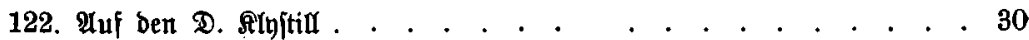

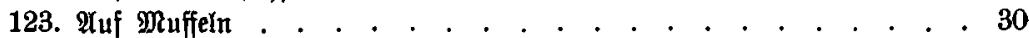

124. $\mathfrak{A n}$ ein $\mathfrak{B a a r}$ arme verwaijete $\mathfrak{M a ̈ b d j e n t ~ . ~ . ~ . ~ . ~ . ~ . ~ . ~ . ~ . ~ . ~ . ~ . ~} 30$

125. $\mathfrak{A} \mathfrak{n}$ ben $\mathfrak{B a x}$. . . . . . . . . . . . . . . . . . . 30

126. Aluf ben (Entharijt . . . . . . . . . . . . . . . . . . . 31

127. Der befte $\mathfrak{W u r f}$. An ein $\mathfrak{\text { Bar }}$ Brettipieler. . . . . . . . . . 31

128. $\mathfrak{A}$ f Den Maler STefós . . . . . . . . . . . . . . . . . 31

129. $\mathfrak{A} u f$ einen Btwenfampf . . . . . . . . . . . . . . . . . 31

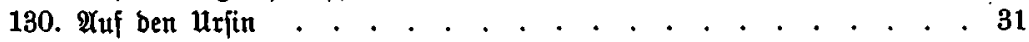

131. צuf ben Beit . . . . . . . . . . . . . . . . . . . . 32

132. Die Borfpiele ber ßerjöhnung . . . . . . . . . . . . . . 32

133. Uuf ben 囚friem . . . . . . . . . . . . . . . . . . . 32

134. Яuf den $\mathfrak{X}$ bar . . . . . . . . . . . . . . . . . . . . 32

135. Seufzer eitte Sranten . . . . . . . . . . . . . . . . . 33

136. Uuf den $\mathfrak{R a a r}$. . . . . . . . . . . . . . . . . . . . 33

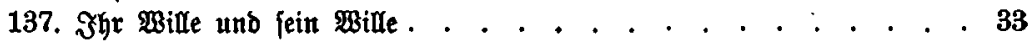

138. Grabiarift ber Tohter eines Freunbes, bie vor ber Taufe ftarb . . 33

139. Xuf ben Marius . . . . . . . . . . . . . . . . . . . 33

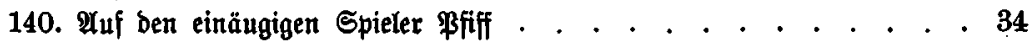

141. $\mathfrak{A n}$ einen $\mathfrak{A}$ utor . . . . . . . . . . . . . . . . . . . 34

142. थuf bett $\mathfrak{R e y}$. . . . . . . . . . . . . . . . . . . . 34

143. Die Sinngebidhte über fíd felbft. . . . . . . . . . . . . . 34

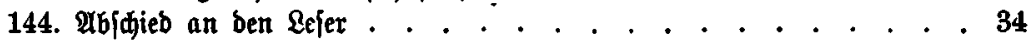

$9 \mathfrak{A} \mathfrak{h} \mathbb{a} \boldsymbol{g}$.

I. Sinngebidte aus ben Sळrifften 1753 , bie 1771 nidht wieber abgebrudt wurben.

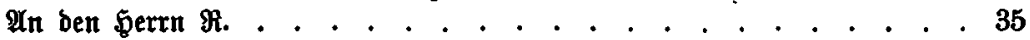

Auf einent befanntert Didter. . . . . . . . . . . . . . . . . 35

Der Bwang . . . . . . . . . . . . . . . . . . . . . . 35

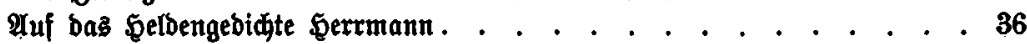


Heโprả山 . . . . . . . . . . . . . . . . . . . 36

Turan . . . . . . . . . . . . . . . . . . . 36

Sertor . . . . . . . . . . . . . . . . . . . . . 36

श्Un Den Dorilas . . . . . . . . . . . . . . . . . . 36

2Uff bie Theftylis . . . . . . . . . . . . . . . . . . . . 37

2uf ben Sophron . . . . . . . . . . . . . . . . . . . . 37

Madahmung besి 84ten Sinngebidts im 3ten Buđje bes Martials . . . . 37

Iuf bas Bebidt bie Sündfluth. . . . . . . . . . . . . . . . 37

Iuf ben urban . . . . . . . . . . . . . . . . . . . . 37

Charlotte. . . . . . . . . . . . . . . . . . . . 38

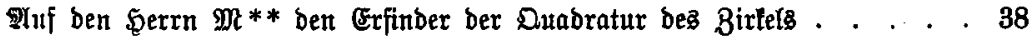

Aluf einen elenden tomijhen Didhter . . . . . . . . . . . . . . 38

$\mathscr{U} \mathfrak{u} \mathfrak{f}==s$. . . . . . . . . . . . . . . . . . . 39

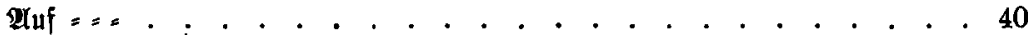

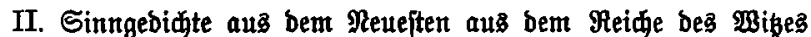
นnঠ auz ber Berfinifden Beitung, bie weber 1753 nod 1771 mieber abgebructt rurben.

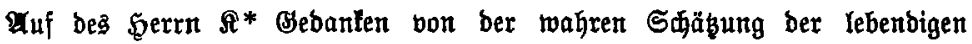
Sräfte. . . . . . . . . . . . . . . . . . . . . 41

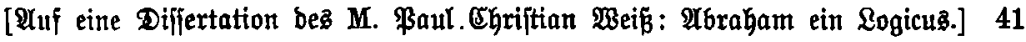

Cin anbers . . . . . . . . . . . . . . . . . . . . . . 41

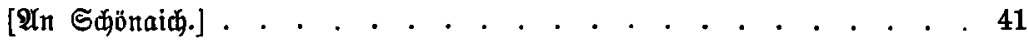

Untwort auf bie Frage: wer $i$ ft ber groffe Dung?. . . . . . . . 42

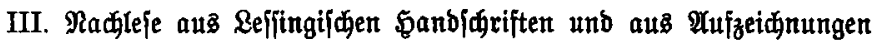
ber Beitgenoffen.

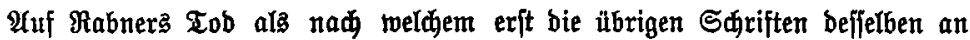
bas sidjt tommen follen . . . . . . . . . . . . . . 42

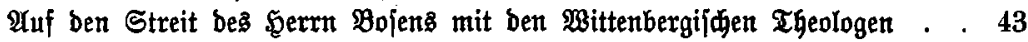

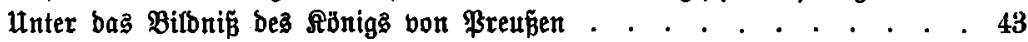

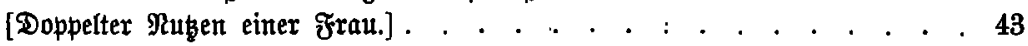

Üuf ein Earuffell . . . . . . . . . . . . . . . . . . . . 43

[Nugen eines fernen Barten.] . . . . . . . . . . . . . . . . 44

Der Blinbe . . . . . . . . . . . . . . . . . . . . . . 44

Sutrz und Sitz. . . . . . . . . . . . . . . . . . . . . . 44

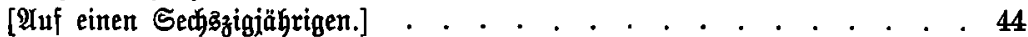

[Der Urme.] . . . . . . . . . . . . . . . . . . . . . 45

$\mathfrak{A} \mathfrak{n}$ Den Dümm . . . . . . . . . . . . . . . . . . . . . 45

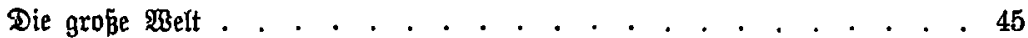

§n ein Stammbuh . . . . . . . . . . . . . . . . . . . 45

Die Berleumbung . . . . . . . . . . . . . . . . . . . . 45 


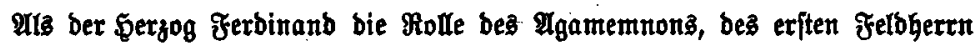
ber \&riechen, fpielte. (1-3) . . . . . . . . . . . . . 45

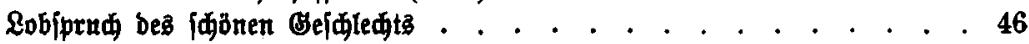

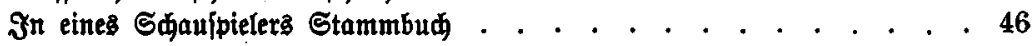

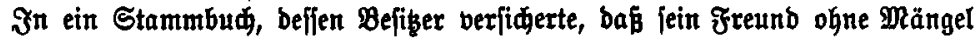
unb fein Mäbden ein Engel fei . . . . . . . . . . . . 47

[æBarum i⿱

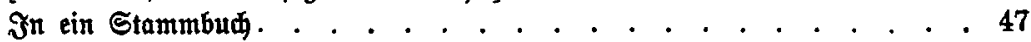

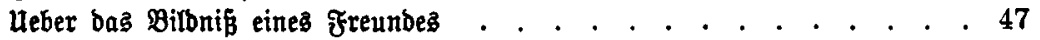

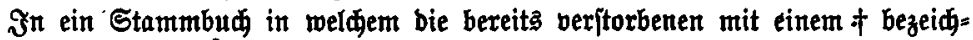
net waren . . . . . . . . . . . . . . . . . . . 48

Sitteriprud . . . . . . . . . . . . . . . . . . . . . 48

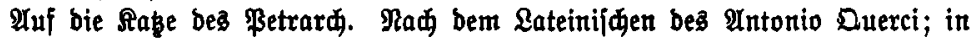
ben Inscriptionibus agri Pataviani . . . . . . . . . . . . 48

Sitteniprud . . . . . . . . . . . . . . . . . . . . . 48

(Grabjhrift auf Boltairen. . . . . . . . . . . . . . . . . 49

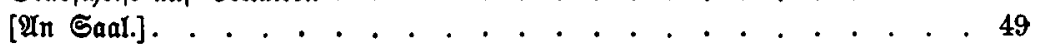

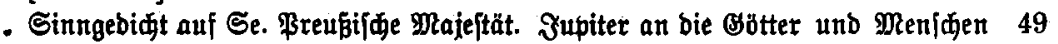

श्uf bas $\mathfrak{U}$ fter . . . . . . . . . . . . . . . . . . . . . 50

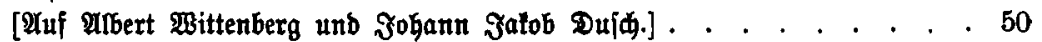

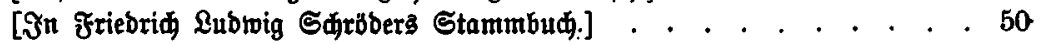

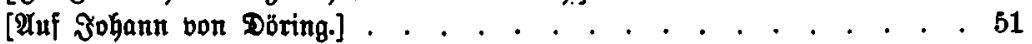

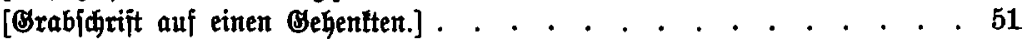

[@rabjarift auf ßteift.] . . . . . . . . . . . . . . . . . . 51

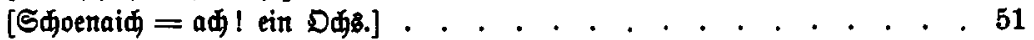

[unbollenbeter Entwurf eines Sinngebiđts..] . . . . . . . . . . . 52

Epigrammata.

Ad Turanium . . . . . . . . . . . . . . . . . . . 55

Ad Gelliam . . . . . . . . . . . . . . . . . . . . 55

In Aristum . ... . . . . . . . . . . . . . . . . . . 55

$\Delta d$ Amicum . . . . . . . . . . . . . . . . . . . . . . 55

Ad Ponticum . . . . . . . . . . . . . . . . . . . . 56

Ad Pompillam . . . . . . . . . . . . . . . . . . . . . 56

In Cæcilianum . . . . . . . . . . . . . . . . . . . . . 56

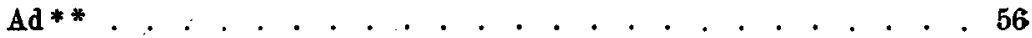

In Albam . . . . . . . . . . . . . . . . . . . . . . . 56

Ad Priseum . . . . . . . . . . . . . . . . . . 56

In Paulum . . . . . . . . . . . . . . . . . 56

Ad Sosibianum . . . . . . . . . . . . . . . . . . . . 57

Ad Posthumum . . . . . . . . . . . . . . . . . . . . 57

Ad Tuccam, ludimagistrum. . . . . . . . . . . . . . . . . 57 


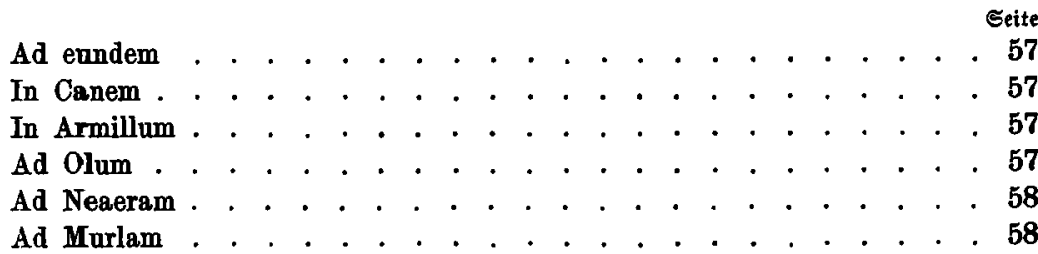

$\mathfrak{x} \mathfrak{n} \mathfrak{h} \mathfrak{n} \mathfrak{g}$.

Ad Naevolam

sieber.

Un bie leber . . . . . . . . . . . . . . . . . . 61

Die Ramen . . . . . . . . . . . . . . . . . . . . . . 62

Die Ruffe . . . . . . . . . . . . . . . . . . . . . . 62

Die Cewí̈beit . . . . . . . . . . . . . . . . . . . . . 63

Die Betribntij. Der Diøter unb jein Freunb . . . . . . . . . . 64

Yntwort eines trunthen Diđtersి . . . . . . . . . . . . . . . 64

Das aufgejobene Gebot . . . . . . . . . . . . . . . . 65

Die Berebjamleit . . . . . . . . . . . . . . . . . . . . 66

Die \$aub̆altung . . . . . . . . . . . . . . . . . . . 66

Der Regen . . . . . . . . . . . . . . . . . . . . . 67

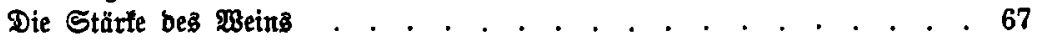

Der Sonberting. . . . . . . . . . . . . . . . . . . . . 67

Der alte unb ber junge Wein . . . . . . . . . . . . . . 68

Die Türten . . . . . . . . . . . . . . . . . . . . . . 68

Mretanber . . . . . . . . . . . . . . . . . . . . 68

Die Sぬöne von Ginten . . . . . . . . . . . . . . . . . . 69

An eine Heine Sujöne . . . . . . . . . . . . . . . . . . 69

Na出 ber 15. Dbe Unatreons . . . . . . . . . . . . . . . . 70

Då ßarabies . . . . . . . . . . . . . . . . . . . . 71

Die Bejpenjter . . . . . . . . . . . . . . . . . . . . . 71

Der truntne Diđter lobt ben Bein . . . . . . . . . . . . . . 73

Sob ber faulfheit . . . . . . . . . . . . . . . . . . . . 74

Die Jaulfheit . . . . . . . . . . . . . . . . . . . . . . 74

Die ßlanetenbetoogner . . . . . . . . . . . . . . . . 75

Der Cefumact ber Mlten . . . . . . . . . . . . . . . . . . 76

Die lügenhafte \$htllis . . . . . . . . . . . . . . . . . . 76

Die fieben unb biersigite Dbe Anatreons . . . . . . . . . . . . 77

Radjałmung biejer Dbe . . . . . . . . . . . . . . . . . . 77

Dex æunj ... . . . . . . . . . . . . . . . . 77

Der gropjte Mann . . . . . . . . . . . . . . . . . . 78

Der §rrthum . . . . . . . . . . . . . . . . . . . . 79 


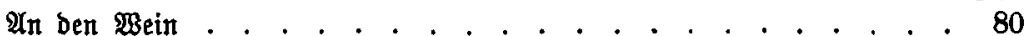

ßhyllis an Damon . . . . . . . . . . . . . . . . . . . 80

Für wen id finge. . . . . . . . . . . . . . . . . . . 81

Die fallafende Laura. . . . . . . . . . . . . . . 82

Der Dontrer. . . . . . . . . . . . . . . . . . . . . 83

Der multäge ßöbel . . . . . . . . . . . . . . . . . . . 84

Die Mufil . . . . . . . . . . . . . . . . . . . . 84

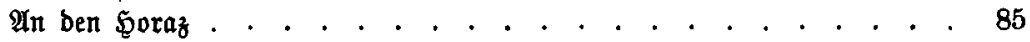

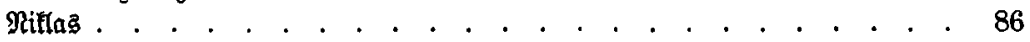

Die Rủffe. . . . . . . . . . . . . . . . . 86

Der ¡đürende Riebhaber . . . . . . . . . . . . . . . . . 86

Trinflieb . . . . . . . . . . . . . . . . . . . . . . 87

Der Berlujt . . . . . . . . . . . . . . . . . . . . . . 87

Der Getú́ . . . . . . . . . . . . . . . . . . . . . 88

Das \&eben . . . . . . . . . . . . . . . . . . . 88

Die Biene . . . . . . . . . . . . . . . . . . . . . . 89

Die \&iebe. . . . . . . . . . . . . . . . . . . . . . 89

Der Tob . . . . . . . . . . . . . . . . . . . . . . 90

Der Faule . . . . . . . . . . . . . . . . . . . . . . 91

Der Flohr . . . . . . . . . . . . . . . . . . . . . . 92

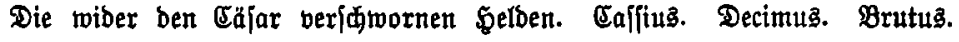

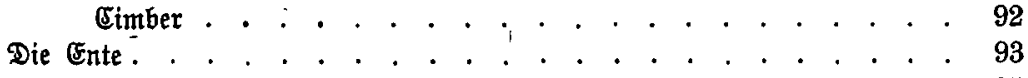

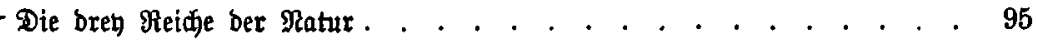

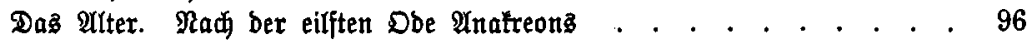

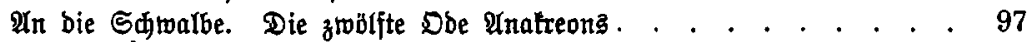

Die đunftridter und ber Diđter. . . . . . . . . . . . . . . 97

Art bie Munftrifuter . . . . . . . . . . . . . . . . . . . 98

\section{'थ $\mathfrak{A} \mathfrak{h a n g . ~}$}

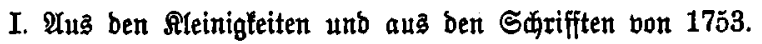

Die berjulimmerte Beiten . . . . . . . . . . . . . . . . . 99

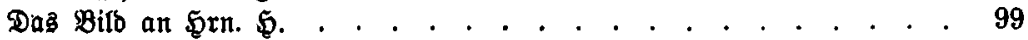

Das $\mathfrak{u m}$ medjeln . . . . . . . . . . . . . . . . . . . . 100

Der Better und bie Mufme . . . . . . . . . . . . . . . . 100

Die Muttex. . . . . . . . . . . . . . . . . . . . 101

Die $\mathfrak{A n t w o r t ~ . ~ . ~ . ~ . ~ . ~ . ~ . ~ . ~ . ~ . ~ . ~ . ~ . ~ . ~ . ~ . ~ . ~ . ~} 101$

Der Słlaf . . . . . . . . . . . . . . . . . . 102

Det philofophifád Trinfer . . . . . . . . . . . . . . . 103

Der Fehler . . . . . . . . . . . . . . . . . . . . 103

Bhnllis lobt den Mein . . . . . . . . . . . . . . . . . . 104

În ben Irnatreon . . . . . . . . . . . . . . . . . 105 
Seite

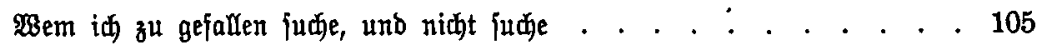

Das Erbbeben . . . . . . . . . . . . . . . . . . . . 109

Die Einwohner bes Mondes . . . . . . . . . . . . . . . . 110

Dex Taufh all $\mathfrak{T r}$. $\mathfrak{B}$. . . . . . . . . . . . . . . . . . . 111

II. $\mathfrak{A}$ us ben Âleinigfeiten.

Die Sparjamfeit . . . . . . . . . . . . . . . . . . . 112

Die $\mathfrak{A b t w e d j a n g ~ . ~ . ~ . ~ . ~ . ~ . ~ . ~ . ~ . ~ . ~ . ~ . ~ . ~ . ~ . ~ . ~ . ~ . ~} 113$

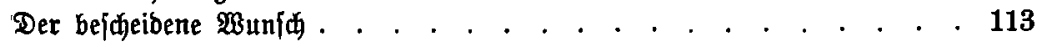

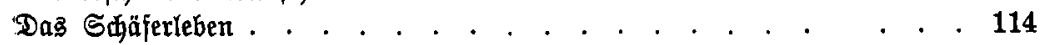

Salomon . . . . . . . . . . . . . . . . . . . . . . . 115

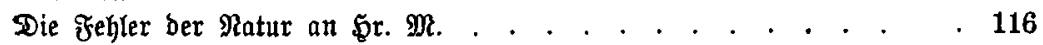

Die fđlimmite Frau . . . . . . . . . . . . . . . . . . . 116

Der Edjiffudf. . . . . . . . . . . . . . . . . . . . . 117

Die Reblichfeit . . . . . . . . . . . . . . . . . . 118

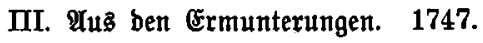

Der Tabat . . . . . . . . . . . . . . . . . . . . . . 119

Refutatio Papatus . . . . . . . . . . . . . . . . . . . 120

IV. $\mathfrak{A}$ us bem Raturforidher. 1747. 1748.

Die 2 Setterprophecebung . . . . . . . . . . . . . . . . 121

Der Sommer. . . . . . . . . . . . . . . . . . . . . 122

Der Gandel. . . . . . . . . . . . . . . . . . . . . . 122

Die Berfteinerung. . . . . . . . . . . . . . . . . . . . 123

Die lehrende $\mathfrak{A}$ trontomie . . . . . . . . . . . . . . . . . 124

V. Raditeje.

Süben und Trinten . . . . . . . . . . . . . . . . . . 126

थuf fid fellgft . . . . . . . . . . . . . . . . . . . . 126

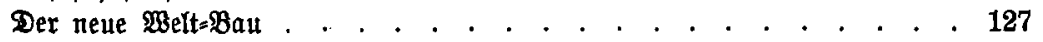

$\mathfrak{A} \mathfrak{n} \mathfrak{A m b r}_{\mathbf{m}} . . \quad$. . . . . . . . . . . . . . . . . . . 127

\$hillis . . . . . . . . . . . . . . . . . . . . . . . 128

\&ieb . . . . . . . . . . . . . . . . . . . 128

Die Diebir . . . . . . . . . . . . . . . . . . . . . 129

Qieb aus bem Spanifhen . . . . . . . . . . . . . . . . . . . . . 129

Eelbenlieb Der Spartaner . . . . . . . . . . . . . . . . . . 129

§ळ. . . . . . . . . . . . . . . . . . . . . . . . . 131

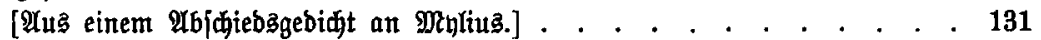

Eine Bejunbbeit . . . . . . . . . . . . . . . . . . . 132

Doen.

I. Der CFintritt bes 1752jten Jahres . . . . . . . . . . . . . 135

ПI. $\mathfrak{A}$ f eine vornehme Bermăhlung $\quad$. . . . . . . . . . . . . . . . 136 


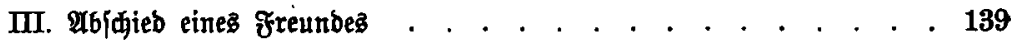

IV. $\mathfrak{A}_{\mathfrak{n}}$ ben நerrn $\mathfrak{R} * *$. . . . . . . . . . . . . . . . . . . 140

V. Der Tob eines Greunbes . . . . . . . . . . . . . . . 141

VI. Der Eintritt bes Эahres 1753. in Berlin . . . . . . . . . 143

VII. Der 24jte Senner in Bertin . . . . . . . . . . . . . . 144

VIII. $\mathfrak{A n}_{\mathfrak{n}}$ jeinen Bruber . . . . . . . . . . . . . . . . . 145

IX. Der Eintritt bes צabres 1754. in Berlin . . . . . . . . . 146

$\mathfrak{A} \mathfrak{n} \mathfrak{h} \mathfrak{n} \mathfrak{g}$.

[Der Eintritt bes $\mathfrak{J a h r e s ~} 1755$ in $\mathfrak{B e r f i n . ] ~ . ~ . ~ . ~ . ~ . ~ . ~ . ~ . ~ . ~ . ~ . ~} 148$

[थ̈n Măcen.]. . . . . . . . . . . . . . . . . . . . . . 149

Drpheus . . . . . . . . . . . . . . . . . . . . . . . 150

$\mathfrak{U} \mathfrak{n}$ Đerr Gleim . . . . . . . . . . . . . . . . . . . . . 150

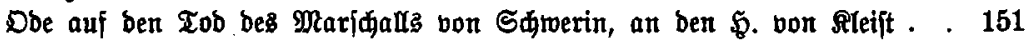

[Ueberfeşung ber Obe beş foraz ad Barinen.] . . . . . . . . . . 153

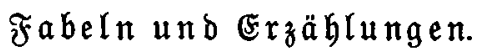

I. Der Sperling und bie Gelbmaus. . . . . . . . . . . . , 157

II. Der פXler unb bie Eule . . . . . . . . . . . . . . . . 157

III. Der Tanzbär . . . . . . . . . . . . . . . . . . . 158

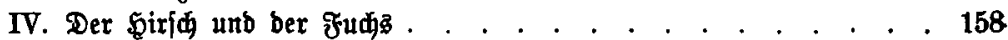

V. Die Sonne. . . . . . . . . . . . . . . . . . . . . 159

VI. Das Mufter ber Egen . . . . . . . . . . . . . . . . 161

VII. Fauftin . . . . . . . . . . . . . . . . . . . . . 161

VIII. Die ehelide Liebe. . . . . . . . . . . . . . . . . 162

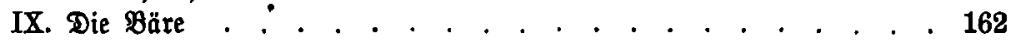

X. Der \&ötwe und bie Mtữe . . . . . . . . . . . . . . . 164

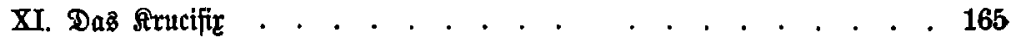

XII. Der Eremit . . . . . . . . . . . . . . . . . . . . 166

XIII. Die Brille . . . . . . . . . . . . . . . . . . . . 177

XIV. Nix Bobentrom . . . . . . . . : . . . . . . . . . 179

\section{$\mathfrak{A} \boldsymbol{n} \mathfrak{h} \mathfrak{\pi g}$.}

I. $\mathfrak{A}$ uล ben Sđdrifften 1753.

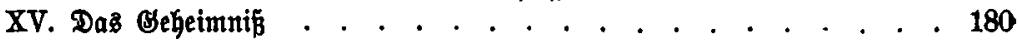

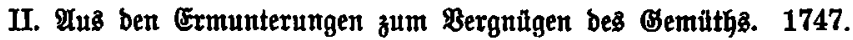

Der 2 unj zu fterben . . . . . . . . . . . . . . . . . . 182

Frene Uleberję̧ung einer Erzählung aus bem Fontaine. Die trante ßulderia 186

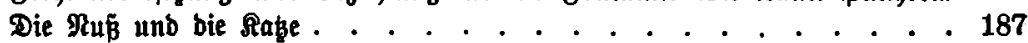

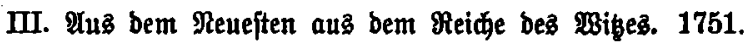

Moryban . . . . . . . . . . . . . . . . . . 188 
IV. Nagiteje.

Seite

Die Teilung .

$\mathfrak{F} \mathfrak{a} \mathfrak{b} \mathfrak{e l n}$.

Drey $B$ ü山er.

Erites Bud.

I. Die Erideinung . . . . . . . . . . . . . . . . . 195

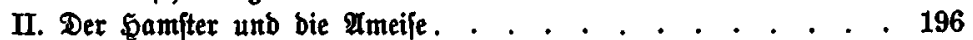

III. Der \&b̆we unb ber $\mathfrak{s a j e}$. . . . . . . . . . . . . . . 196

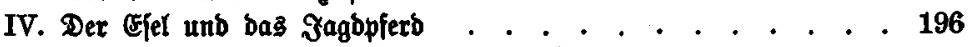

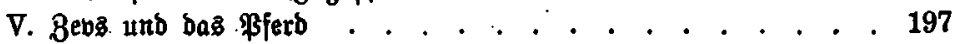

VI. Der $\mathfrak{A f f e}$ und ber fuds . . . . . . . . . . . . . . 197

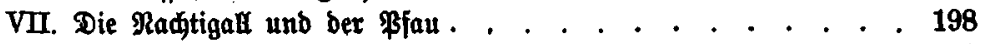

VIII. Der 280 off unb ber Sđáfer . . . . . . . . . . . . . 198

IX. Das Roß unb ber Stier . . . . . . . . . . . . . . 198

X. Die Grifle unb bie Radtigall . . . . . . . . . . . . . 199

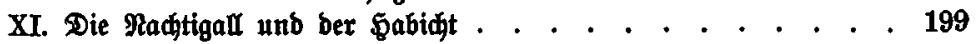

XII. Der triegerifde Bolf . . . . . . . . . . . . . . . 199

XIII. Der \$gonnix . . . . . . . . . . . . . . . . . . 200

XIV. Die હans . . . . . . . . . . . . . . . . . . . 200

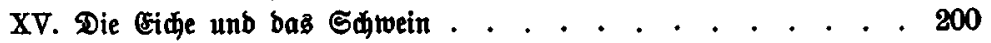

XVI. Die ßespen . . . . . . . . . . . . . . . . . . 201

XVII. Die Sperlinge. . . . . . . . . . . . . . . . . . 201

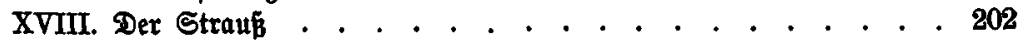

XIX. Der Eperling utto ber Straús . . . . . . . . . . . 202

XX. Die פunbe . . . . . . . . . . . . . . . . . . . 202

XXI. Dex Frudis und ber Stord . . . . . . . . . . . . . 203

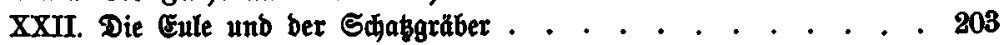

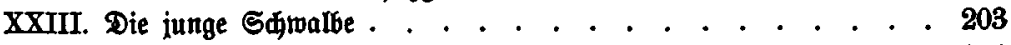

XXIV. Merops . . . . . . . . . . . . . . . . . . . . 204

XXV. Der \$elelelan . . . . . . . . . . . . . . . . . 204

XXVI. Der Lôte unb der Tieger. . . . . . . . . . . . . . 205

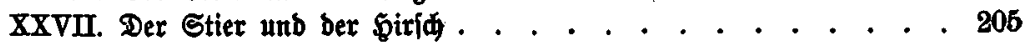

XXVIII. Der Gfet unb ber 280 lf . . . . . . . . . . . . 205

XXIX. Der Springer im Sdjadje. . . . . . . . . . . . 206

XXX. Aejopus unb ber Ejel . . . . . . . . . . . . . 206

Brwentes Bud.

I. Die ełgerne Bilblăule . . . . . . . . . . . . . . . 206

II. Sertules . . . . . . . . . . . . . . . . . . . . 207

III. Der §nabe und die ভdjlange. . . . . . . . . . . . 207 
IV. Dex $\mathfrak{B o l f}$ auf bem Tobtbette. . . . . . . . . . . . . 208

V. Der Stier und bas §alb. . . . . . . . . . . . . . 208

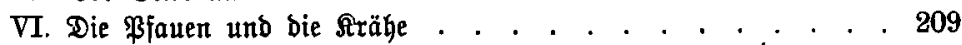

VII. Der \&üme mit bem Ejel . . . . . . . . . . . . . . 209

VIII. Der Efel mit bem Sömen. . . . . . . . . . . . . . 209

IX. Die blinbe ఏenne . . . . . . . . . . . . . . . . 210

X. Die Ejel . . . . . . . . . . . . . . . . . . . 210

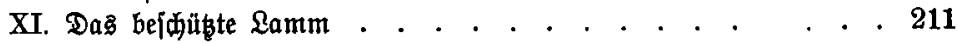

XII. Jupiter und $\mathfrak{A p o l l o ~ . ~ . ~ . ~ . ~ . ~ . ~ . ~ . ~ . ~ . ~ . ~ . ~ . ~ . ~ . ~ . ~} 211$

XIII. Die Wafferiflange . . . . . . . . . . . . . . . . 211

XIV. Der Fuch und bie Rarbe. . . . . . . . . . . . . . 212

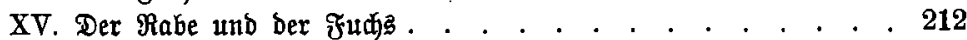

XVI. Der Beibige . . . . . . . . . . . . . . . . . . 213

XVI. Der Rabe. . . . . . . . . . . . . . . . . . . 213

XVIII. Вebs unb bas ভ丸jaf . . . . . . . . . . . . . . . 213

XIX. Der Fuđ̆ und ber Tieger . . . . . . . . . . . . . 214

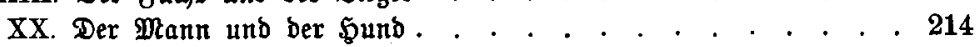

XXI. Die Traube . . . . . . . . . . . . . . . . . 215

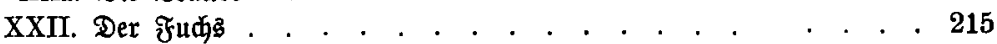

XXIII. Daş కfaf . . . . . . . . . . . . . . . . . . 215

XXIV. Die Biegen . . . . . . . . . . . . . . . . . . . 216

XXV. Der wilbe $\mathfrak{2}$ pfelbaum . . . . . . . . . . . . . . . 216

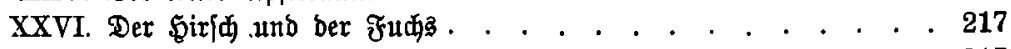

XXVII. Der Dornjtraúf . . . . . . . . . . . . . . . 217

XXVIII. Die Furien. . . . . . . . . . . . . . . . . . . 217

XXIX. Tirefias . . . . . . . . . . . . . . . . . . . . 218

XXX. Minerba . . . . . . . . . . . . . . . . . . . . 218

Drittes $\mathfrak{B u d}$.

I. Der Befizer bę̧ Bogenż . . . . . . . . . . . . . . 219

II. Die Radtigall und bie Rerdje . . . . . . . . . . . . . . . 219

III. Der Seift beas Salomo. . . . . . . . . . . . . . . 219

IV. Das Gejhenf ber Fenen . . . . . . . . . . . . . . 220

V. Das Shaf und bie Shtwalbe . . . . . . . . . . . . 220

VI. Der $\Re$ abe . . . . . . . . . . . . . . . . . . . 221

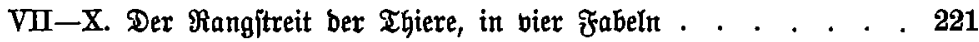

XI. Der $\mathfrak{B a ̈ r}$ unb ber Elephant . . . . . . . . . . . . . 222

XII. Der Straú̃ . . . . . . . . . . . . . . . . . . 223

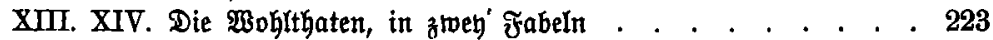

XV. Die Eidie . . . . . . . . . . . . . . . . . . . 224

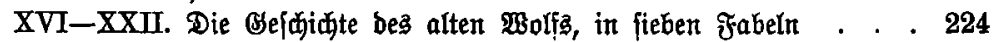

XXIII. Die $\mathfrak{M a u}$. . . . . . . . . . . . . . . . . . . 227 
Seite

XXIV. Die Sdmalbe . . . . . . . . . . . . . . . . 228

XXV. Dex Яrolet . . . . . . . . . . . . . . . . . . . 228

XXVI. Der junge unb ber alte ફirid . . . . . . . . . . . . 228

XXVII. Der $\mathfrak{B f a u}$ unb ber $\mathfrak{b a h n ~ . ~ . ~ . ~ . ~ . ~ . ~ . ~ . ~ . ~ . ~ . ~ . ~ . ~ . ~} 229$

XXVIII. Dex நirí . . . . . . . . . . . . . . . . . . . 229

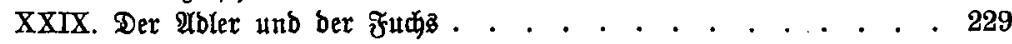

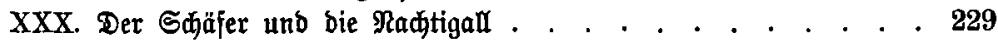

$\mathfrak{A} \mathfrak{n} \mathfrak{h} \mathfrak{a} \mathfrak{n}$.

I. Fabeln aus ben Sđarifften 1753 , bie 1759 und 1777 niđt wieber abgebrutt wurben.

Der Rieje . . . . . . . . . . . . . . . . . . . 230

Der Falfe . . . . . . . . . . . . . . . . . . . . . 230

Damon und Theodor . . . . . . . . . . . . . . . . . . 231

II. Fabeln, bie erft nadi Leffing Tobe befannt wurben.

Der Sdäferjtab . . . . . . . . . . . . . . . . . . . . 232

Der Maturalift . . . . . . . . . . . . . . . . . . . . . 233

Der Wolf unb bas Săf . . . . . . . . . . . . . . . . 234

$\mathfrak{F} \mathfrak{r} \mathfrak{a} \mathfrak{g} \mathfrak{m} \mathfrak{n} \mathfrak{t} e$.

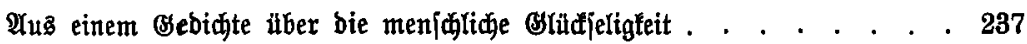

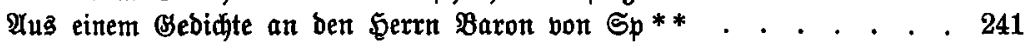

Yus einem Bebidte über ben jebigen Brefdmat in ber Poefie . . . . . 242

Uus eirem Bebidte an ben $\mathfrak{b e r r n} \mathfrak{M} * *$. . . . . . . . . . . . . 243

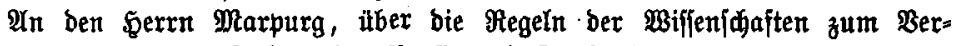
gnügen; bejonderb der Poefie unt Tonfunft. . . . . . . . . 248

Die Meligion. Erjter Bejang. . . . . . . . . . . . . . . 255

$$
\mathfrak{A} \mathfrak{n} \mathfrak{h a n} \mathfrak{g} .
$$

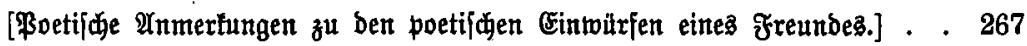

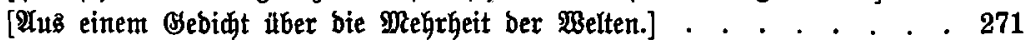

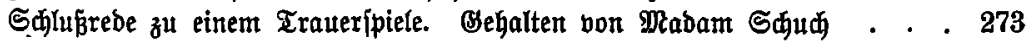

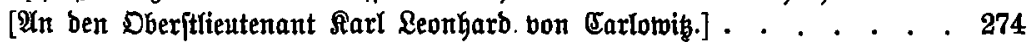

Der junge Gelehrte. Cin Suttpiel in bren $\mathfrak{A}$ ufzügen . . . . . 279

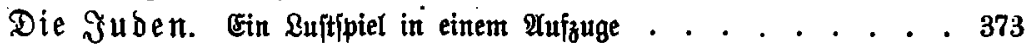


